\title{
Shaping the science-industry-policy interface in synthetic biology
}

\author{
Sibylle Gaisser $\cdot$ Thomas Reiss
}

Received: 11 May 2009/Revised: 22 June 2009/Accepted: 25 June 2009

(C) The Author(s) 2009. This article is published with open access at Springerlink.com

\begin{abstract}
Current advances in the emerging field of synthetic biology and the improvements in key technologies promise great impacts, not only on future scientific development, but also on the economy. In this paper we will adopt the triple helix concept for analyzing the early stages of a new field of science and innovation, namely synthetic biology. Synthetic biology is based on the creation and assembly of parts in order to create new and more complex structures and functions. These features of synthetic biology raise questions related to standardization and intellectual property, but also to security and public perception issues that go beyond the classical biotechnology discussions. These issues concern all involved actors in the synthetic biology field and affect the interrelationship between science, industry and policy. Based on the results of the recently finished EU FP-6 funded project TESSY (http://www.tessy-europe.de), the article analyzes these issues. Additionally, it illustrates the setting of clear framework conditions for synthetic biology research and development and the identification and definition of common goals for the future development of the field which will be needed for efficient science-industry-policy interaction. It was shown that it will be crucial to develop approaches that consider the needs of science and industry, on the one hand, and comply with the expectations of society, on the other hand. As synthetic biology is a global activity, the involvement of national decision-makers in
\end{abstract}

\section{S. Gaisser $(\bowtie) \cdot$ T. Reiss}

Fraunhofer Institute Systems and Innovation Research,

Breslauer Str. 48, 76139 Karlsruhe, Germany

e-mail: sibylle.gaisser@isi.fraunhofer.de

T. Reiss

e-mail: thomas.reiss@isi.fraunhofer.de

URL: www.isi.fraunhofer.de international initiatives will further stimulate the development of the field.

Keywords Standardization - Intellectual property rights . Security · Public involvement · Framework conditions . Knowledge transfer

\section{Introduction}

The importance of efficient interactions between scienceindustry and government has been discussed among innovation research scholars for about 15 years. Leydersdorff and Etzkowitz (1996) coined the term "triple helix of university-industry-government relations" to describe a new mode of understanding innovation processes where the efficient interplay between these three institutions becomes a main driving force of innovation. According to this perspective, academia, or more broadly speaking, public research organizations, industry and government collaborate to create new knowledge, technology, processes and products which are finally transmitted to users for final usage or for interim use to produce other goods or services. An important difference between triple helix innovation and traditional collaborative processes between academia, industry, and government is that in the triple helix case all three institutions are playing fully integrated and overlapping roles. The triple helix framework has developed into a powerful analytical concept in particular for explaining a new role of universities in innovation processes. The concept states that universities can play an enhanced role in innovation in the increasingly knowledge-based societies (Etzkowitz and Leydesdorff 2000).

In this paper we will adopt the triple helix concept for analyzing the early stages of a new field of science and 
innovation, namely synthetic biology. We will elaborate that even at very early stages of emerging disciplines the interrelation between the three triple helix components plays an important role for setting the scene for further development of the field. We also argue that adopting the triple helix concept for emerging technologies already at very early stages could help to overcome some important roadblocks for generating triple helix innovation dynamics. Such roadblocks include

- diverging or even conflicting attitudes towards intellectual property (strong interest of industry in protection versus a culture of free exchange and a strong interest in publishing among academia);

- differing expectations concerning benefits from collaborative projects (basic research activities supporting gain in academic reputation versus focused applied approach towards commercial application and expected financial returns);

- different perspectives with respect to time horizons of activities;

- mainly disciplinary organisation of academic research due to, e.g., disciplinary oriented career schemes opposed to interdisciplinary industrial perspective and experience driven by the need to solving problems using the best suited mix of disciplines or technologies.

Synthetic biology is based on the creation and assembly of parts in order to create new and more complex structures and functions. Although one could argue that also classical biotechnology and metabolic engineering aimed at creating something new, synthetic biology adds a new quality to this approach. Whereas classical biotechnology aimed at exploiting biological systems, living organisms or parts of them in order to enlarge the knowledge base, produce goods or provide services, synthetic biology aims at providing biological parts and contributing to the de-novo synthesis of non-natural biological systems for a variety of applications and/or the understanding of the origin of life. The modularity of parts and the availability of orthogonal systems provide a new level of abstraction to bio-engineering. These features of synthetic biology immediately raise questions related to standardization and intellectual property. In addition, since new devices that did not exist at all as such in nature may also exert unforeseen or even dangerous functions, security and public perception issues need to be considered. All theses issues concern all involved actors in the synthetic biology field and in particular the interrelation between science, industry and policy is addressed. Based on results of a recently finished EU FP-6 funded project, TESSY (http://www.tessy-europe. de), we will discuss these issues from a triple helix perspective. In addition we will also consider two other areas which will need efficient science-industry-policy interaction. Theses are the setting of clear framework conditions for synthetic biology research and development and the identification and definition of common goals for the future development of the field.

\section{Issues for science-industry-policy interaction in synthetic biology}

Framework conditions

From the very beginning of synthetic biology research the community discussed whether the field of synthetic biology is the logical extension of classical genetic engineering or whether it has completely new features. Deciding in favour of the one or the other has consequences for the regulatory framework. Assuming that synthetic biology is a further development of biotechnology, the regulatory model of rDNA biotechnology should be appropriate. However, a recent analysis of the Woodrow Wilson International Center for Scholars showed that there are indeed new challenges such as the question of risk assessment. In a context in which the parent agent (chassis, DNA) is less obvious, classical risk assessment strategies cannot be applied any more (Rodemeyer 2009). "The framing process for synthetic biology is now well underway and the outcome will depend on the thoughtful engagement of all interested parties" (Rodemeyer 2009). Initiatives such as the Joint Conference of the OECD, the UK Royal Society and the US National Academies of Science on "Opportunities and Challenges in the Emerging Field of Synthetic Biology" 9-10 July 2009, Washington DC are supposed to be a basis for such a dialogue among all stakeholder groups. The development of an appropriate regulatory framework for synthetic biology may be considered as a key task to be accomplished at the interface between science, industry and governmental agencies. This also implies that the regulatory framework for synthetic biology could be finally considered as a proof of concept concerning a functional triple helix system.

\section{Standardization}

Industry representatives in particular argue that synthetic biology can only develop its benefits and will find its way into industrial research and development if parts and devices are standardized and clearly characterized. According to this view, the availability of "off the shelf" biological bricks needs to be supported by structured fact sheets of the specificity of a biological compound as is already in place for a number of parts and devices that are collected in the "Registry of Standard Biological Parts" (http://partsregistry.org/ Main_Page). But standardization has to go beyond physical 
assembly standards and experimental and measurement standards. Though in general scientists are rather reluctant if their freedom of research is limited, as may be the case through standards, synthetic biologists now agree that standards in reporting, organizing, storing, and sharing biological parts could facilitate their own work. This could finally lead to computer/human readable formats for parts and visual formats. In Table 1, an overview of currently discussed topics in standardization for synthetic biology is given. In this sense standardization could be a facilitator to establish efficient triple helix alliances.

It seems obvious that standardization cannot be invented by any (political) institution or organization in a top-down approach, but has to be developed by the users (academia and industry) themselves. In the initiative "Provisional BioBrick Language" (PoBol; http://www.pobol.org), it is planned to capture the minimal information needed to describe a BioBrick, while remaining extensible to additional data and open for interlinking. The project is thought to involve the scientific community as is indicated by its name "pobol" which is also the Welsh word for people. Therefore, the founders around Raik Grünberg and Jason Morrison suggest regular 2 day meetings and portable software libraries to enable developers to incorporate capabilities into other software.

Within the TESSY project (http://www.tessy-europe.eu), a roadmap for the future development of the field in Europe was elaborated, covering not only scientific and technological milestones but also considering important framework conditions such as funding, regulation, and public perception (Gaisser et al. 2009a). One milestone for the next 8 years will be the development of common standards in synthetic biology. The roadmap suggests adopting an approach with four consecutive steps: (1) standards for reporting until 2010, (2) standards for methods and components until 2012, (3) a general standard for all -omics approaches until 2014 and (4) standards for the underlying mathematics until 2016. The scope of steps number 3 and 4 is not yet completely clear and can be defined in more detail once scientific development in synthetic biology and the basic standardization of parts and devices is further advanced. This clearly shows the need for the suggested bottom-up approach and for intensive interactions among the scientific community, industry and national or international governmental institutions which are responsible for standardization.

\section{Intellectual property issues}

The feature of synthetic biology is to share and reuse standard biological parts and devices. Traditionally it is well established among the scientific community to exchange vectors, strains and other items on a bilateral

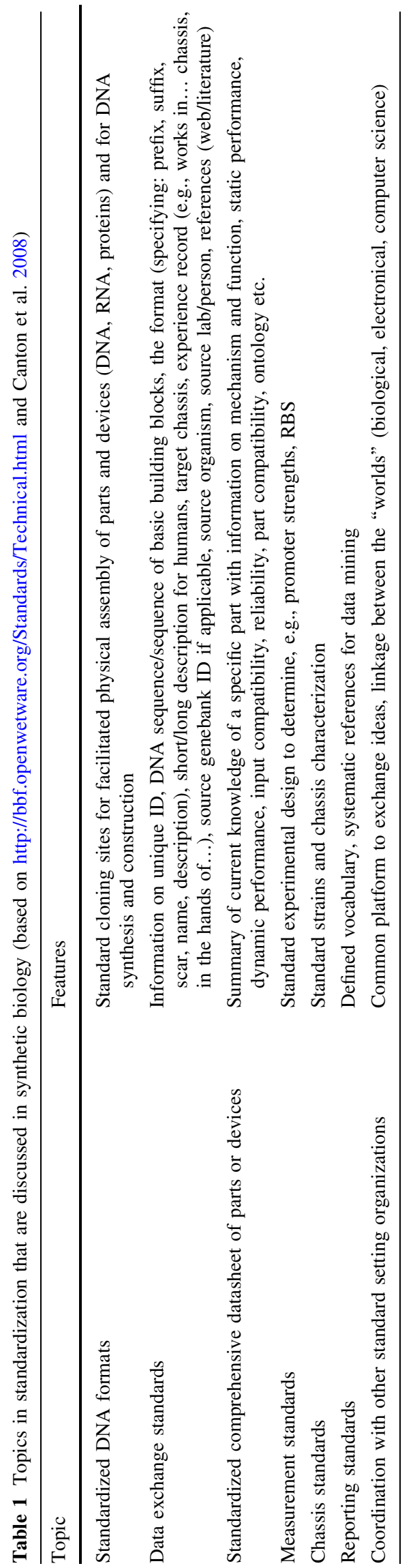


basis. For industry, however, sharing items seems to be an unrealistic position at the present time. As long as the donor of materials cannot guarantee freedom to operate, it is assumed that the IP practice of industry is not likely to change for some time. Additionally, it can be argued that industry would not contribute to a real system of openness as there are tendencies to maintain a two-tiered system, keeping the highly valuable parts locked up. The different cultures and interests in intellectual property issues between industry and academia seem to be a strong roadblock towards efficient triple helix alliances.

The new quality in synthetic biology is that this sharing of parts is mediated via specific registries such as the "Standard Registry of Biological Parts". However, there are some hurdles associated with the construction of such a registry as genes or functional parts are partially already patented and thus cannot be distributed freely among the scientific community. A quick search in the Standard Registry of Biological Parts showed for example that it contains entries that are based on the green fluorescent protein, the TET operator or the T7 promotor. All three constructs are subject to European or global patents. Whether the parts in the database actually fall under this patent protection has to be checked individually. This simple example indicates a certain lack of knowledge among scientists of possibly associated patent issues. A similar situation was recently described in the field of molecular diagnostics, where laboratory directors who used patented tests had only limited awareness of the actual legal status of the test or denied the applicability of patents to gene tests (Gaisser et al. 2009b).

The unclear patent situation creates a feeling of uneasiness among scientists, as became evident during the participatory roadmapping process in the TESSY project. The clarification of the open source status of biological parts and the international harmonization of IP issues were assessed to be highly relevant. A clarification within a short term perspective was called for.

International organizations already started to deal with the subject, but currently there seems to be a long way to go. Until then, IP issues remain to be solved on an individual basis according to current law. This implies that each scientist has to be aware of IP issues. He or she has to identify possible patent holders of parts by patent search or through public calls as outlined by Berthold Rutz, patent officer at the European Patent Office (personal communication). The next steps will be to negotiate non-assertion for research purposes and indicate parts covered by patents and status (asserted/non-asserted, unknown) in database. It will be necessary to develop license conditions for newly generated parts based on patent rights and/or patent pool(s) for synthetic biology. As detailed knowledge of intellectual property rights is required, the collaboration of scientists with well informed technology transfer offices and the IP departments of private companies will be required. It may be also helpful to establish public institutions for support and information in IP questions, as has been shown in other areas. Efficient cooperation between science, industry, and governmental institutions will be necessary.

\section{Security aspects}

Security threats, in the sense of the intentional release of biological agents, play an important role in the discussion between policy and synthetic biology associated industry. In particular, the DNA synthesis sector is concerned, since ordered DNA fragments could also bear the danger of being misused so that basically a careful screening and checking of all orders including customers would be required. Both policy-makers, namely the national Ministries of Foreign Affairs, and the private sector will be asked to come up with measures and international agreements to prevent misuse. The security concerns are not new to synthetic biology. Biotechnology and even microbiology also had to deal with these concerns of misuse. Against this background there are scientists who argue that there is no specific security risk in synthetic biology. On the other hand, the DNA synthesis industry is confronted with the questions of how to deal with potentially harmful DNA orders or clients from potentially aggressive states.

In a first initiative, the International Association of Synthetic Biology (IASB; http://www.ia-sb.eu) developed suggestions for technical solutions for biosecurity in synthetic biology. This refers to a comprehensive screening of ordered DNA fragments and their clients. The IASB members agreed in their meeting in April 2008 to create a wiki-style non-public forum to discuss shortcomings and possible improvements in screening software and to share technical resources on a non-competitive basis. It was decided that IASB will cooperate with the Goldman School of Public Policy in building the infrastructure for a virulence factor database. This resource, the "Virulence Factor Information Repository-VIREP" will be a web-based, publicly accessible database containing the annotated genomes of selected viruses, bacteria and possibly eukaryotic pathogens. Finally, the Industry Association decided to closely interact with national and international decision-makers to support efforts to create international policies.

This initiative is a first step towards achieving an important milestone outlined in the TESSY roadmap on regulation that highlighted the need for the development of clear guidance for research and development until 2009. Further milestones include a code of conduct until 2011 and the implementation of measures to prevent misuse until 2013. 
Involvement of the public

Lessons from other emerging fields such as green biotechnology showed that transparency regarding goals and realistic communication of scientific results are crucial to create an atmosphere of openness and trust. Although communication with the public is not a guarantee that the public will accept all scientific goals, it is helpful to identify and shape research priorities (Hampel and Renn 1999). Measures and timing of public involvement are the subject of intense discussions. As democratic societies want to ensure that the public can make informed decisions and engage in the public dialogue on science policy, intime information of the public seems to be essential. On the other hand, there is the risk of communicating unrealistic promises, thereby raising expectations which will hardly be met by reality (Balmer and Martin 2008).

A second problem is the size of the targeted groups and the degree of details. In smaller countries such as Switzerland or Denmark it may be possible to engage comprehensively in public dialogues. Bigger countries may apply Internet-based approaches such as the 1,000 questions campaign in Germany on bioethics (http://www.1000 fragen.de). In any case there is still a need to develop, apply and evaluate suitable methods and approaches for efficient public dialogue schemes.

The TESSY roadmap on synthetic biology also indicates the need for public involvement. Both "educational activities on all levels" and "raise public awareness" were assessed as continuous activities that should start immediately. It is worth mentioning that during the participatory process within the context of the development of the TESSY roadmap experts estimated that $5-10 \%$ of the funding budgets for synthetic biology should be dedicated to ELSA research and accompanying measures.

\section{Definition of common goals}

Judging from some recent international conferences on synthetic biology, the field is still addressing/faced with a number of basic research questions. On the other hand, industry has a strong interest in applications either in order to develop new products or to apply the synthetic biology approach to their own research and development. In order to further promote the field, it seems essential to intensify the discussion between stakeholders about joint research efforts. A prominent example of such joint efforts is the collaboration of public and private institutions and companies in developing the malaria drug Artemisinin. In fact, the development of a semi-synthetic artemisinin production system by the introduction of a number of metabolic gene clusters in a production strain is described as one of the first examples for the proof of the principle of synthetic biology though there are scientists who classify this approach still as metabolic engineering rather than synthetic biology. Partners are the Institute for One World Health, which leads the product development and the responsibility for directing the collaborative effort; additionally it leads the project's public policy and global access goals; Amyris Biotechnology Inc, which is responsible for optimizing the microbial strain and using it in the development of a manufacturing process to make high quality semisynthetic artemisinin; Sanofi-aventis, which provides fermentation and chemistry process development expertise, and if technical benchmarks are achieved, will be responsible for the development of an industrial manufacturing process for semi-synthetic artemisinin and the University of California at Berkeley, which has utilized synthetic biology to develop a microbial strain to produce artemisinic acid. The University of California at Berkeley completed its portion of the development efforts in December 2007 (source: http://www.artemisininproject. org/partners). It is worth mentioning that from the triple helix perspective the Artemisinin example is not a perfect case since the commercial partners act on a non-profit basis. However, it shows that especially in emerging technologies alternative funding models and the support of non-profitorganizations and foundations may be an essential step towards paving the way for future industrial uptake of the technology. In particular it illustrates how the interaction between triple helix partners generates a strong innovation dynamics. In Europe we are also observing first efforts to establish such new public-private partnerships. Examples include the EU funded projects Emergence (http://www. emergence.ethz.ch) and TARPOL (http://www.sb-tarpol.eu).

\section{Conclusions}

In this contribution we discuss a number of important socioeconomic issues related to synthetic biology research which are directly relevant for the science-industry-policy interface. In order to advance activities related to standardization, intellectual property, security, and public perception it seems crucial to jointly develop approaches which consider the needs of science and industry, on the one hand, but also comply with the expectations of society on the other hand. Such joint efforts will also contribute to creating an atmosphere of openness and dialogue among all stakeholders. Obviously, the role of governmental institutions or more generally the role of policy could be to act as a moderator in the dialogue between science and industry. In addition, an important policy task already in these very early stages would be to support activities aiming at communicating synthetic biology research issues to the public. Results of TESSY research where the scientific community called for significant public funding of ELSA research related to synthetic biology strongly support this policy task. 
Synthetic biology is a global activity. Accordingly, national efforts related to standards, intellectual property or security issues are not sufficient and need to be complemented by international initiatives. This means that national decision-makers need to engage in respective international activities. Recent examples include international working groups such as the OECD Synthetic Biology Steering Group or the Knowledge-based Bio-Economy (KBBE-NET) Collaborative Working Group on synthetic biology.

Until now there have been only a few highlight projects such as the production of the malaria drug Artemisinin which demonstrate the potential of the synthetic biology approach. However, this example illustrates that efficient collaboration between science and industry is a key to success. It seems crucial that science and industry collaborate to provide proof of the principle of the synthetic biology approach. An obvious role for policy would be to facilitate and to support such new public private partnerships. Thereby the strong dynamics of triple helix type innovation could be utilized, already in early stages of the new field of synthetic biology and could exert a significant push to advancing the field.

Open Access This article is distributed under the terms of the Creative Commons Attribution Noncommercial License which permits any noncommercial use, distribution, and reproduction in any medium, provided the original author(s) and source are credited.

\section{References}

Balmer A, Martin P (2008) Synthetic biology social and ethical challenges. http://www.bbsrc.ac.uk/organisation/policies/reviews/ scientific_areas/ 0806_synthetic_biology.pdf

Canton B, Labno A, Endy D (2008) Refinement and standardization of synthetic biological parts and devices. Nat Biotechnol 26(7):787-793

Etzkowitz H, Leydesdorff L (2000) The dynamics of innovation: from national systems and "Mode 2" to a triple helix of universityindustry-government relations. Res Policy 29:109-123

Gaisser S, Reiss T, Lunkes A, Müller KM, Bernauer H (2009a) Making the most of synthetic biology - strategies for synthetic biology development in Europe. EMBO Rep 10(special issue): $\mathrm{S} 1-4$

Gaisser S, Hopkins MM, Liddell K, Zika E, Ibarreta D (2009b) The phantom menace of gene patents. Nature 458:407-408

Hampel J, Renn O (eds) (1999) Gentechnik in der Öffentlichkeit: Wahrnehmung und Bewertung einer umstrittenen Technologie. Verlag Campus, Frankfurt

Leydesdorff L, Etzkowitz H (1996) Emergence of a triple helix of university-industry-government relations. Sci Public Policy 23:279-286

Rodemeyer M (2009) New life, old bottles. Synbio2, March 2009. http://www.synbioproject.org/library/publications/archive/syn bio2/ 